\title{
PERCEPCIONES ASOCIADAS AL MÉTODO MADRE CANGURO DE MADRES CON RECIÉN NACIDOS PREMATUROS
}

\author{
PERCEPTIONS ASSOCIATED WITH THE KANGAROO MOTHER \\ METHOD OF MOTHERS WITH PREMATURE NEWBORNS
}

\section{PERCEPÇÓES ASSOCIADAS AO MÉTODO MÃE CANGURU DE MÃES COM RECÉM-NASCIDOS PREMATUROS}

\author{
Carmen Gloria Barraza Peña* \\ Viviane Eugenia Jofré Aravena** \\ Jonathan Andrés Ramírez Ocho****
}

\begin{abstract}
RESUMEN
Objetivos: El presente estudio buscó conocer las percepciones de madres de recién nacidos prematuros, asociadas a la condición de prematuridad, cuidado en incubadora y uso del Método Madre Canguro. Material y Método: Se realizó un estudio en un con metodología cualitativa, entre julio y diciembre 2015, mediante estudio de casos, basado en la Teoría Fundamentada de Glaser y Corbin usando el Método de Comparación Constante. El muestreo fue de tipo intencional u opinático constituido por 22 díadas: madre y recién nacido prematuro de la Unidad de Paciente Crítico Pediátrico de un hospital al sur de Chile. Para el análisis, se usó codificación abierta, axial y selectiva, limitando la muestra en función del criterio de saturación teórica. Resultados: El recién nacido prematuro, repercute emocionalmente en la madre generando sentimientos negativos, siendo estos propiciados por la hospitalización y los cuidados en la incubadora. El Método Madre Canguro es un factor protector en la emocionalidad positiva de la madre, favoreciendo su alegría, tranquilidad, vínculo afectivo temprano y empoderamiento. Conclusiones: El Método Madre Canguro ayuda al desarrollo del recién nacido prematuro y mejora el estado emocional de la madre frente a una situación adversa, contribuyendo, paralelamente, al bienestar del recién nacido.
\end{abstract}

Palabras clave: Método Madre-Canguro; Recién Nacido; Recién Nacido Prematuro; Enfermería; Investigación cualitativa.

\footnotetext{
*Enfermera, Psicóloga Clínica, Magíster en Educación para las Ciencias de la Salud. Departamento Materno Infantil, Facultad de Enfermería, Universidad de Concepción, Concepción Chile. ORCID: https://orcid.org/0000-0002-7398-1984 Email: cbarraza@ udec.cl

**Enfermera, Doctora en Enfermería. Departamento Materno Infantil, Facultad de Enfermería, Universidad de Concepción. Concepción, Chile. ORCID: https://orcid.org/0000-0002-9192-6145 Email: vjofre@udec.cl Autora de correspondencia

***Enfermero, Magíster en Enfermería. Departamento Materno Infantil, Facultad de Enfermería, Universidad de Concepción. Concepción, Chile. ORCID: https://orcid.org/0000-0002-0682-2115 Email: jonaramirez@udec.cl
} 


\section{ABSTRACT}

Objective: The following study aims at presenting the perceptions of mothers of premature newborns, associated with the condition of prematurity, incubator care, and use of the Kangaroo Mother Care Method. Material and Method: The study was carried out with a qualitative approach, between July and December 2015, through case studies based on the Grounded Theory of Glaser and Corbin, using the Constant Comparative Method. The sampling was purposive and consisted of 22 dyads: mother and premature newborn of the Unit of Pediatric Critical Patient of a hospital in the south of Chile. For the analysis, open, axial and selective coding was used, limiting the sample according to the theoretical saturation criterion. Results: The premature newborn baby affects the mother emotionally, causing negative feelings, which are influenced by hospitalization and incubator care. The Kangaroo Mother Method is a protective factor in the positive emotionality of the mother, favoring her happiness, tranquility, early affective bond and empowerment. Conclusions: The Kangaroo Mother Method helps the development of the premature newborn and improves the emotional state of the mother in the face of an adverse situation, contributing at the same time to the well-being of the newborn baby.

Key words: Kangaroo Mother Care Method; Newborn; Premature Newborn; Nursing; Qualitative Research.

\section{RESUMO}

Objetivos: O presente estudo buscou conhecer as percepçóes de mães de recém-nascidos prematuros, associadas à condição de prematuridade, cuidados em incubadora e utilização do Método Mãe Canguru. Material e Método: Foi realizado um estudo com metodologia qualitativa, entre julho e dezembro de 2015, por meio de um estudo de caso, baseado na Teoria Fundamentada de Glaser e Corbin utilizando o Método da Comparação Constante. A amostra foi do tipo intencional ou baseada na opinião, composta por 22 díades: máe e recémnascido prematuro da Unidade de Paciente Crítico Pediátrico de um hospital do sul do Chile. Para a análise, utilizou-se a codificação aberta, axial e seletiva, limitando a amostra de acordo com o critério de saturação teórica. Resultados: $\mathrm{O}$ recém-nascido prematuro causa impacto emocional na mãe, gerando sentimentos negativos, estes sendo causados pela internação e os cuidados na incubadora. O Método Mãe Canguru é um fator protetor na emocionalidade positiva da mãe, favorecendo sua felicidade, tranquilidade, vínculo emocional precoce e empoderamento. Conclusóes: O Método Mãe Canguru auxilia no desenvolvimento do recém-nascido prematuro e melhora o estado emocional da mãe diante de uma situação adversa, contribuindo, paralelamente, para o bem-estar do recém-nascido.

Palavras-chave: Método Mãe-Canguru; Recém-nascido; Recém-nascido prematuro; Enfermagem; Pesquisa qualitativa.

Fecha de recepción: 12/12/2019

Fecha de aceptación: 16/04/2020

\section{INTRODUCCIÓN}

El recién nacido prematuro (RNPT) se define como aquel resultante de un embarazo que finaliza espontáneamente o por indicación médica antes de las 37 semanas de gestación; los casos que se presentan previo a las 34 semanas representan un 60 a $70 \%$ del total de la morbi-mortalidad perinatal $^{(1)}$. Debido a la situación anterior y la tasa global de fecundidad nacional de Chile (1,83 hijos por mujer), los programas de salud han priorizado la atención del recién nacido (RN) desde la gestación hasta la incorporación en el sistema escolar ${ }^{(2)}$.

El Método Madre Canguro (MMC) se define como el contacto piel a piel entre madre e hijo/a de forma precoz y continua; el niño viste pañal, calcetines y gorro; colocándose en posición fetal contra el pecho materno, buscando evocar su vida 
intrauterina y favoreciendo la lactancia materna ${ }^{(3,4)}$. La técnica canguro nace en Bogotá, Colombia, el año 1978 , como respuesta al hacinamiento y falta de incubadoras. En el año 1998, la United Nations International Children's Emergency Fund (UNICEF) declara el MMC como eficaz y revolucionario para salvar vidas, basado en el amor, el calor y la leche materna, acunándose desde entonces el concepto "incubadoras humanas" para referirse a la relación madre-hijo en el $\mathrm{MMC}^{(5)}$.

La evidencia describe que el MMC es eficaz en la reducción de infecciones asociadas a la atención en salud, estabilización de parámetros hemodinámicos y termorregulación del RNPT, además de disminuir el estrés de la madre y empoderarla como proveedora principal de las necesidades físicas y emocionales, estableciendo un vínculo afectivo único e indisoluble entre la madre y el RNPT ${ }^{(6-10)}$.

En el contexto nacional de Chile, todavía existe una brecha en el conocimiento respecto al impacto de la aplicación del MMC sobre la madre, aun cuando desde el programa Chile Crece Contigo se da énfasis en su participación en diferentes actividades con el fin de favorecer el empoderamiento del cuidado y el fortalecimiento de las relaciones vinculares ${ }^{(2)}$.

La Teoría del Apego y la Teoría de la Regulación identifican que para el RN es un periodo sensible dado por el proceso de maduración y desarrollo neuronal, donde el encuentro afectivo entre madre y RNPT es capaz de regular en forma inconsciente activadores fisiológicos y emocionales en la madre, favoreciendo la adquisición de habilidades y mecanismos que permiten enfrentar y regular el estrés, las emociones, el aprendizaje y los estados cognitivos, afectivos y conductuales en el proceso de desarrollo del RNPT ${ }^{(11-13)}$.

La modulación cerebral del RNPT y la madre están dadas por acciones como: mirarse a los ojos, jugar, tocarse, emitir expresiones faciales y cambiar de posturas corporales, entre otras, generando un lenguaje sin palabras, donde la díada se comunica y adapta funciones afectivas, fisiológicas y $\operatorname{cognitivas}^{(14)}$; no es un proceso universal, porque es mediado por las diferencias individuales y por un ambiente vincular facilitador.

La madre actúa como soporte relacional, calmando o activando los estados del RN a través de la sensibilidad, entendiéndose ésta como la habilidad para percibir las señales del niño, interpretarlas y responder a ellas de manera adecuada y contingente. De esta forma, actuaría como un organizador externo en la regulación bio-conductual del niño, desarrollando funciones intelectuales superiores del lóbulo frontal, como la resolución de problemas, toma de decisiones y en algunos casos, previniendo cuadros como el déficit atencional y concluyendo, por lo tanto, que el cerebro es un órgano psicosocial ${ }^{(15-18)}$.

Es significativo entonces, estudiar el MMC como vivencia de las madres, sean estas: emociones, sentimientos y/o percepciones. La sensibilidad y sincronía a responder de forma oportuna a las necesidades del niño y la estimulación cognitiva durante los primeros ańos de vida, se relacionan con la calidad del medio familiar como clima proclive a las relaciones afectivas continuas y coherentes, de tal forma que el apego y el cerebro del niño se conviertan en co-dependientes que, en consecuencia, genera un cuidado eficaz, al reducir el comportamiento intrusivo del niño y favorecer un apego seguro ${ }^{(17,19-21)}$. En Chile, el MMC está contemplado como estrategia de intervención temprana sobre los factores de riesgo modificables para mejorar la calidad de vida de los padres y sus hijos, aun sin extenderse hegemónicamente al servicio público ${ }^{(2)}$.

Considerando la importancia del MMC y la necesidad de revelar la experiencia en torno a éste, el objetivo de la presente investigación fue conocer las percepciones de madres de RNPT, asociadas a la condición de prematuridad, cuidado en incubadora y la implementación del MMC. Se espera que los resultados permitan desarrollar y fundamentar planes de atención de enfermería que reconozcan a la madre como el eslabón principal del cuidado del RNPT.

\section{MATERIAL Y MÉTODO}

Se realizó un estudio con metodología cualitativa durante el ańo 2015, mediante estudio de casos, basado en la Teoría Fundamentada de Strauss y Corbin usando el Método de Comparación Constante $^{(22)}$, replanteado por Uwe Flick ${ }^{(23)}$, que permite la ampliación de la teoría del apego y el reconocimiento de los lazos vinculares entre la madre y el RNPT. 
Para el análisis se usó codificación abierta, axial y selectiva, limitando la muestra en función del criterio de saturación teórica con la totalidad de la información recopilada, lo que admite expresar los datos e información surgida en forma de conceptos, para después agruparlos en categorías mayores y relevantes para la pregunta de investigación e identificando las categorías y subcategorías que nacen de los dominios relatados en las entrevistas durante la investigación.

Los criterios de rigor, validez y confiabilidad de la investigación cualitativa utilizados fueron los de credibilidad, auditabilidad y transferibilidad. Lo anterior se realizó mediante la triangulación en la recolección de los datos y en el análisis posterior de los datos, mediante una entrevista previa a la aplicación de las grabaciones de audio y video de 60 min por visita durante la implementación del MMC, facilitando así la expresión de sentimientos emergentes. Posteriormente se revisaron las grabaciones de audio - video, se hizo transcripción de entrevistas y revisión de notas de campo, buscando la congruencia en los resultados y análisis.

La unidad de paciente crítico pediátrico del hospital está compuesta por una unidad de cuidados intensivos (UCI), unidad de cuidados intermedios (UTI) y unidad de cuidados mínimos, con cuatro, seis y doce cupos respectivamente, con horario de visita entre las 12:00 a 18:00 h, tiempo que puede ser ajustado dependiendo de las necesidades individuales del servicio.

Si bien la aplicación del MMC se encuentra normada, no siempre es posible su aplicación debido a la sobrecarga laboral y emergencias que se pueden presentar fortuitamente. Los investigadores se desempeñan como enfermeros docentes en clínica de neonatología y pediatría, con estudiantes de $3^{\circ}$ y $4^{\circ}$ año de Enfermería de una Universidad. Con la aceptación del Comité Ético Científico del hospital ( $\left.\mathrm{N}^{\circ} 2379\right)$, y la autorización de la Dirección de la Unidad, se comenzó el trabajo de campo, entre los meses de julio a diciembre del 2015, iniciando con una revisión de las fichas clínicas de RNPT hospitalizados, que se extendió por dos semanas, previa aplicación de criterios de inclusión: prematuridad (32 a 34 semanas de gestación), peso mayor a 1500 gramos, estabilidad del patrón respiratorio, reflejo de succión y deglución presentes y/o susceptibles de ser estimulados, hijo/a de padres chilenos, visitados por la madre y embarazo único. Se seleccionó una muestra total de tipo intencional u opinático, formada inicialmente por 4 diadas. Como resultado del proceso analítico de la codificación abierta, se obtuvo una lista de categorías y subcategorías emergentes, que se fueron codificando según los significados que las madres expresaron durante la aplicación del MMC. Al cabo de seis meses, al ser homogéneas, es decir, cuando no emergieron nuevas categorías de análisis, se logró saturar la muestra con un total de 22 diadas finales. Simultáneamente, se realizó un acercamiento a las madres en horarios de visita para informar sobre los objetivos y duración del estudio, solicitando la autorización y participación por medio de un consentimiento informado donde se estableció la confidencialidad con nombre ficticio/ edad para la transcripción de las entrevistas.

La totalidad de las madres seleccionadas accedieron a participar en el desarrollo del presente estudio, reconociendo el empoderamiento que tuvo para ellas en la atención de sus hijos RNPT. Primero se realizó una orientación respecto a la aplicación del MMC, en la misma unidad, para posteriormente aplicar 15 sesiones de $40 \mathrm{~min}$ del MMC y una entrevista de cierre de $20 \mathrm{~min}$ posterior a cada aplicación.

Durante la aplicación del MMC se cobijó al RNPT vestido con pañal, gorro y calcetines en el torso descubierto de sus madres, buscando permitir el contacto piel a piel y cubiertos con una mantilla de género para resguardar la privacidad de la madre y la termorregulación del RNPT. Durante este proceso y posteriormente, las madres expresaron las diferentes percepciones surgidas en torno al MMC, condición de prematuridad y cuidados en incubadora, en un espacio físico que favoreció la privacidad y la expresión de la subjetividad y emociones vividas. Todo el proceso anteriormente descrito fue registrado en sistema audiovisual y notas de campo, que fueron transcritos y analizados manualmente por los investigadores. Se cruzaron los casos individuales, en función de las categorías, buscando desarrollar una estructura temática que permitiera la elaboración de similitudes y diferencias entre las representaciones de cada participante, buscando identificar las interacciones recíprocas entre cada una de las partes. 


\section{RESULTADOS}

Las madres participantes tenían una edad entre los 20 y 34 años, multíparas, todas profesaban una religión, con pareja estable y todas con escolaridad media incompleta.

La Figura 1 muestra las interacciones recíprocas entre categorías y subcategorías. Estas fueron emergiendo de acuerdo con los dominios que las madres relataron, asignándoles una connotación de acuerdo con el proceso analítico y emocional que vivenciaron durante la aplicación del MMC (Tabla 1). Frente a lo anterior, fue posible identificar las percepciones de las madres respecto a la condición de prematuridad, cuidados en incubadora y la aplicación del MMC, integrando los relatos representativos que forman cada categoría y subcategoría ${ }^{(22,23)}$ :

Percepciones de la condición de prematuridad: Durante el proceso hospitalario, las madres manifestaron escasa comprensión del diagnóstico, frustración en su rol de madre, disminución de la autoestima, temor respecto a si su hijo alcanzaría un correcto desarrollo y sentimientos de culpa asociado al hecho de no poder contribuir en el cuidado por encontrarse a cargo del personal de salud, donde el despliegue de tecnologías y cuidados específicos les asusta y genera temores. Lo anterior fue expresado en frases como:

"Me cuesta imaginar lo que está pasando, ¿será culpa mía?" (Jazmín/26).

"Creo que me equivoque en muchas cosas ¿Sera sano?" (Geranio/22).
"Me siento frustrada y disminuida como mujer $y$ madre" (Gladiolo/35).

Al momento del alta, surge la culpa nuevamente en el relato de las madres, por no haber hecho algo para evitar la prematurez y en muchos casos, no contar con redes de apoyo para continuar con el cuidado en el hogar. Lo anterior se evidenció en frases como:

“Cómo lo haré en la casa?", “iseñorita, usted sabe cuántos días más estará aqui??....si me hubiese cuidado esto no hubiese pasado" (Tulipán/20).

"Reconocerá que yo soy la mamá, si tantas enfermeras lo atienden?" (Crisantemo/21).

Percepciones asociadas al cuidado en incubadora: La definición de esta categoría presenta una connotación negativa para las madres, donde a pesar de identificar que es necesaria para la estadía y bienestar del RNPT, consideran la incubadora como una caja fría con cables, que expone a su hijo/a casi desnudo a las miradas de terceros. Las subcategorías desprendidas fueron culpa, angustia, incertidumbre, impotencia y tristeza.

Las madres relatan que el observar a su hijo/a a través de la incubadora, les provoca impotencia y tristeza, produciéndoles la percepción de que su hijo se encuentra en un ambiente poco natural, una especie de vientre artificial, reforzando su sentimiento de culpa respecto al supuesto de que hizo algo mal durante el embarazo o de que algo está mal en su propia constitución de cuerpo-mujer.

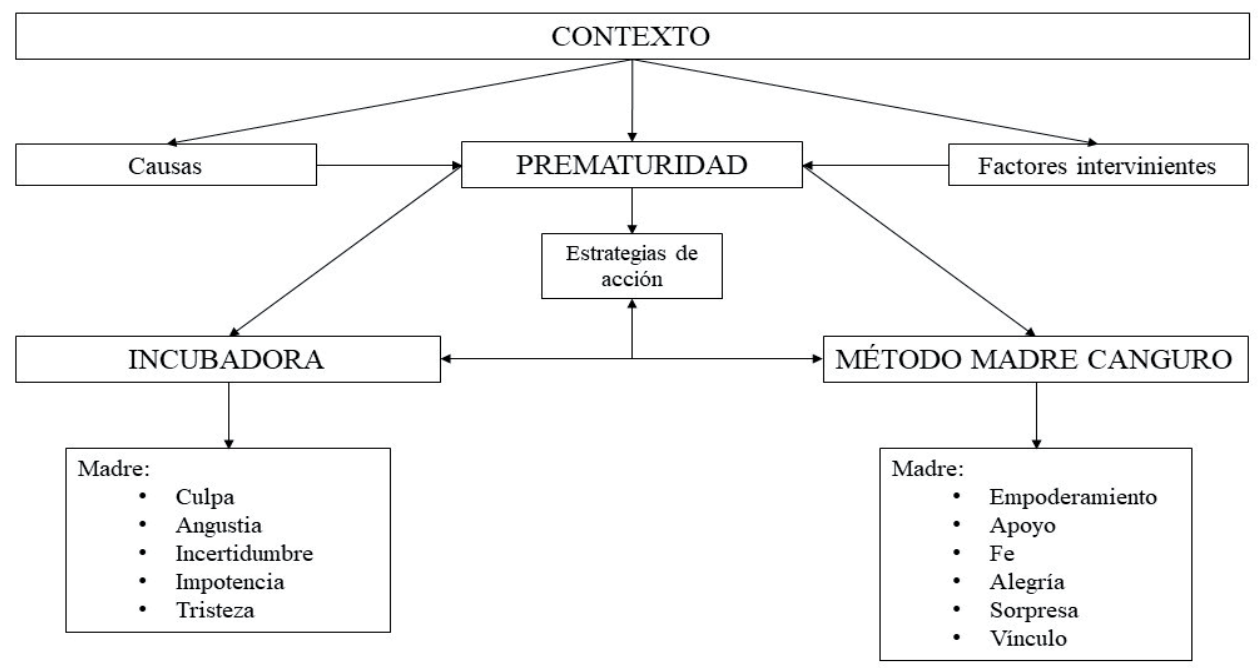

Figura 1. Flujograma de interacciones recíprocas entre categorías y subcategorías. 
Tabla 1. Categorías y subcategorías.

\begin{tabular}{ccl}
\hline Categoría & \multicolumn{1}{c}{ Definición } & \multicolumn{1}{c}{ Subcategorías } \\
\hline $\begin{array}{c}\text { Percepción de la condición de } \\
\text { prematuridad }\end{array}$ & $\begin{array}{c}\text { Grado de conocimiento, causalidad, } \\
\text { temores asociados, días de } \\
\text { hospitalización y vínculo de la diada. }\end{array}$ & $\begin{array}{l}\text { Comprensión del Diagnóstico } \\
\text { Causas identificadas } \\
\text { Temor e inseguridad } \\
\text { Culpa }\end{array}$ \\
\hline $\begin{array}{ccc}\text { Percepción asociada al cuidado en } \\
\text { incubadora }\end{array}$ & $\begin{array}{c}\text { Connotación negativa necesaria para } \\
\text { estadía y bienestar: caja fría que expone } \\
\text { a las miradas }\end{array}$ & $\begin{array}{l}\text { Culpa } \\
\text { Angustia } \\
\text { Incertidumbre } \\
\text { Impotencia }\end{array}$ \\
& & Tristeza \\
\hline Percepción asociada a la & Connotación positiva. Favorece el & Empoderamiento \\
implementación del MMC & desarrollo del RNPT y fortalece el & Apoyo \\
& vínculo de la diada. & Fe \\
& & Alegría \\
& & Sorpresa \\
& & Vínculo \\
\hline
\end{tabular}

La palabra "riesgo", que acompaña al reporte diario sobre el estado de salud del RNPT, aparece como una constante amenaza, lo que contribuye a su angustia, impotencia e incertidumbre, reforzando la idea de un futuro incierto, manifestado en frases como:

"Lo veo tan pequeño e indefenso que no sé si puedo tomarlo" (Lirio/25).

“Será capaz de vivir en esa cajita transparente?, ¿tendrá frio?” (Violeta/27).

"Si yo hubiese tenido más cuidado, tal vez no habría nacido antes de tiempo" (Pensamiento/32).

"...y ¿todos esos cables, son necesarios?...ंno se puede vestir con más ropa?” (Clavel/24).

\section{Percepciones asociadas a la implementación} del MMC: Frente a la implementación del MMC, las madres relataron una experiencia gratificante y enriquecedora al interaccionar y sentirse unida con su hijo/a, aportando felicidad y esperanza a través del contacto piel a piel y favorecido por la lactancia materna efectiva. Se rompía así el círculo de inseguridad, ayudando a controlar y reducir la ansiedad, que favoreció, además, el empoderamiento sobre la lactancia materna y dio tiempo para mayor integración del padre. Recalcan la importancia de compartir la experiencia, proyectándola en el tiempo para incluso ser aplicada en el hogar. Lo anterior fue manifestado en frases como:

"Ah!, igual como los canguritos que se ven en la televisión, no tenía idea que se podía hacer con mi hijo" (Girasol/34).

"Ya no me da miedo que sea tan chiquitito, ahora puedo cambiarle pañales" (Gladiolo/35).

"Sentirlo en mi pecho y poder alimentarlo es maravilloso" (Margarita/22).

"Estando juntitos y al sentir su respiración, es como si fuéramos uno solo" (Crisantemo/21).

Las madres describieron que el apoyo y la calidad de la información entregada por el personal de salud durante la aplicación del MMC y los horarios de visita son primordiales para sobrellevar la angustia e inseguridad, además que las prepara para brindar los cuidados en el hogar permitiendo la participación del padre, lo cual provoca tranquilidad al momento del alta. La religión y la fe son factores importantes para las madres y la unidad neonatal permite, dentro de sus normas, realizar los ritos religiosos como apoyo espiritual. Lo anterior fue manifestado en frases como:

"Es verdad que se puede bautizar a mi hijo" (Violeta/27).

"Le cambié pañales y pude hacerlo, gracias por su ayuda", "reconoce cuando le hablo" (Margarita/22). 


\section{DISCUSIÓN}

Cabe destacar la homogeneidad presentada entre las madres frente a los dominios emergentes, que es concordante con lo señalado por diferentes autores, quienes indican que la prematuridad es una sorpresa inesperada, que merma la formación del vínculo madre-hijo/a, generando sentimientos de incertidumbre, temor y culpa ${ }^{(24,25-27)}$. Esto puede llegar a ser reforzado por la falta de comprensión que poseen las madres respecto al diagnóstico, frustración en su rol y disminución de la autoestima. Lo anterior, de acuerdo con la literatura, pudiese ser atenuado por las redes de apoyo familiar y del personal de salud, reforzado por la accesibilidad y entrega clara y oportuna de información del reporte de salud de su hijo ${ }^{(21,24-26,28-31)}$, equiparable a lo reportado por las madres cuando en ellas disminuyó el estrés de la hospitalización. Aunque no fue explorado en este estudio, se revela además, que el involucramiento del padre se encuentra estrechamente relacionado con un ambiente favorable en el $\mathrm{MMC}^{(30)}$.

La dedicación exclusiva de las madres al cuidado del RNPT, la condición de prematurez y el cuidado en incubadora, significan una carga emocional, traducido en estrés físico y emocional (21, 24-26, 28-31), que fue identificado en este estudio, reportándose como sentimientos de culpa, angustia, incertidumbre respecto a la evolución, impotencia, tristeza, temor e inseguridad.

Se destacan los beneficios descritos en la literatura en la ejecución del MMC, por la facilidad y bajos costos en su implementación, implicando sólo modificar las rutinas y procedimientos en las salas de hospitalización, con un alto impacto al disminuir el estrés asociado a la condición de prematuridad y a los cuidados en incubadora $(17,21,24-27,32-37)$. Esto concuerda con lo descrito en los resultados, cuando se observó que el MMC favoreció en las madres su capacidad de resiliencia, de dejar atrás la emocionalidad negativa inicial y empoderarse para el cuidado del RNPT, favoreciendo el desarrollo del vínculo madre-hijo por el tiempo que pueden pasar juntos. Aun así, es necesario considerar que muchas veces esto puede ser entorpecido por la sobrecarga laboral existente en los diferentes servicios y unidades, sumado a las contingencias que pudiesen surgir en un momento determinado, asociadas a las condiciones orgánicas de los RNPT.

Los beneficios del contacto piel a piel, independiente de la prematuridad, favorecen la relación madre-hijo, aumentan la satisfacción de ser madre y la sensibilidad de responder oportunamente a las necesidades del RNPT ${ }^{(24-26,28,29,38-40)}$, es decir, el MMC tendría un efecto protector, al mejorar la receptividad de las madres hacia sus hijos. La aplicación del MMC mejora la calidad de vida de las madres, por el mejor contacto físico y afectivo entre ellos, además de incentivar el inicio precoz de la lactancia materna, que se asocia significativamente a ausencia de sintomatología depresiva postparto, mayor autoeficacia de las madres para una lactancia exitosa y un destete $\operatorname{tardío}^{(15,21,32,36,38)}$. En este estudio fue reportado mediante la expresión de sentimientos de alegría y fe en el futuro.

El MMC permite que las madres conozcan mejor a su hijo/a, otorgándoles confianza en la tarea de ser madres. De esta forma, sienten alegría de tener a su hijo/a en casa y con la responsabilidad de que ella es la única que puede cuidar bien de éste ${ }^{(24-}$ 26). A pesar de que no se realizó el seguimiento en domicilio respecto a la aplicación del MMC, las madres manifestaron el deseo de continuar con su implementación en el hogar, posterior al alta, debido a que les daba mayor confianza, comodidad y seguridad.

En la revisión bibliográfica realizada por los autores, no se encontraron desventajas o inconvenientes respecto al uso del MMC, identificándose solo beneficios asociados a su implementación.

Como limitaciones estuvo la restricción horaria durante las visitas, asociada a la carga laboral del personal de la unidad, lo cual fue abordado a través de la extensión horaria del tiempo de visitas y flexibilidad horaria de los investigadores. Dado la restricción del número de personas al interior de la unidad, solo fue posible entrevistar a las madres

\section{CONCLUSIÓN}

El MMC permite un trabajo colaborativo y en equipo entre la madre del RNPT y el personal de salud que brinda la atención directa, siendo una estrategia temprana sobre los factores de riesgo modificables que posee la diada madre-hijo/a, que empodera y mejora el estado emocional de la madre 
frente a la condición de prematuridad y el uso de la incubadora. Las madres reconocen al MMC como un medio que favorece el bienestar, crecimiento y desarrollo de su hijo/a.

Reconocida la validez del MMC, debe ser implementado por los diferentes servicios de atención de RNPT e incorporado a las rutinas de atención diaria porque permite entregar un trato humanizado hacia las madres y sus hijos/as.

\section{REFERENCIAS}

1. Ministerio de Salud. Guía Perinatal 2015 [Internet]. Santiago de Chile: Minsal, Sub-secretaría de Salud Pública; 2015 [citado 10 ene 2019] 466 p. Disponible en: https://www.minsal.cl/sites/default/files/files/ GUIA\%20PERINATAL_2015_\%20PARA\%20 PUBLICAR.pdf

2. Chile Crece Contigo. Cuatro años creciendo juntos: Memoria de la Instalación del Sistema de Protección Integral a la Infancia. Chile crece contigo 2006-2010 [Internet]. Santiago de Chile: Mideplan/Minsal 2011 [citado 10 ene 2019]. Disponible en: http://www.crececontigo. gob.cl/wp-content/uploads/2015/10/ChCC_ MEMORIA.pdf

3. Instituto Materno Infantil. Programa Madre Canguro. Historia y consolidación del programa [Internet]. 2003 [citado 14 dic 2018]. Disponible en: http://madrecanguro.tripod.com/ihistoria.htm

4. Conde-Agudelo A, Díaz-Rossello J. Kangaroo mother care to reduce morbidity and mortality in low birthweight infants. Cochrane Database of Systematic Reviews [Internet]. 2016 [citado 8 may 2019]; 8(CD002771). Disponible en: 10.1002/14651858.CD002771.pub4

5. Fondo de Naciones Unidas para la Infancia (UNICEF). Orientación programática de UNI-CEF para el desarrollo de los nińos y las niñas en la primera infancia [Internet]. USA: División de Programas de UNICEF; 2017 [citado 4 may 2019]. 40 p. Disponible en: https:// www.unicef.org/sites/default/files/2018-12/ Programme\%20Guidance\%20for\%20ECD\%20 \%28SPANISH\%29_1.pdf

6. Rengel C y Labajos M. Efecto del contacto piel a piel con padres de recién nacidos por cesárea sobre la lactancia materna y nuevos roles de paternidad. Enferm Docente [Internet]. 2011 [citado 8 may 2019]; (94):8-15. Disponible en: http://www. index- f.com/edocente/94pdf/94-008.pdf
Para próximos estudios, se recomienda la integración de las percepciones de los padres y/o cuidador, además de identificar variables del RNPT como el peso, talla, termorregulación, control de signos vitales, entre otros, porque son influenciados por la aplicación del MMC. Se sugiere además realizar estudios de seguimiento, de tipo longitudinal de RNPT y sus madres, posteriores al alta.

7. Organización Mundial de la Salud. Método madre canguro: guía práctica [Internet]. Ginebra: Departamento de Salud Reproductiva e Investigaciones Conexas; 2004 [citado 15 may 2019]. 63 p. Disponible en: https://apps.who. int/iris/bitstream/handle/10665/43083/9243 590359.pdf; jsessionid=AC9B4F5837013CB 4A35C058D91E8D876? sequence $=1$

8. Martínez T, Damian N. Beneficios del contacto piel a piel precoz en la reanimación neonatal. Enferm Universitaria [Internet]. 2014 [citado 12 may 2019]; 11(2): 61-66. Disponible en: https:// www.sciencedirect.com/science/article/pii/ S1665706314726661

9. Fernández I. Infección nosocomial y método madre canguro. Revisión de evidencias. ENE [Internet]. 2013 [citado 20 may 2019]; 7(3):15. Disponible en: http://ene-enfermeria.org/ojs/ index.php/ENE/article/view/301

10. López-Andaluz J. Método madre canguro: Valoración de enfermería. Publicaciones didácticas [Internet]. 2016 [citado 10 abr 2019]; 72: 337-349. Disponible en: https:/studylib.es/doc/6111558/ metodo-madre-canguro---publicacionesdid $\% \mathrm{C} 3 \%$ Alcticas

11. Collados-Gómez L, Aragonés-Corral B, Contreras-Olivares I, García-Feced E, Vila-Piqueras M. Impacto del cuidado canguro en el estrés del neonato prematuro. Enferm Clínica [Internet]. 2011 [citado 8 abr 2019]; 21(2): 67-124. Disponible en: https://www.elsevier.es/es-revista-enfermeriaclinica-35-articulo-impacto-del-cuidadocanguro-el-S1130862110002093

12. Johnston C, Filion F, Campbell-Yeo M, Goulet C, Bell L, McNaughton K, et al. Enhaneed Kangaroo mother care for heel lance in preterm neonates: a crossover trial. J Perinat [Internet]. 2009 [citado 8 abr 2019]; 29:51-56. Disponible en: https://www. nature.com/articles/jp2008113

13. Feldman R, Weller A, Sirota L, Eidelman AI. Testing a family intervention hypothesis: The 
contribution of mother-infant skin to skin contact (Kangaroo care) to family interaction, proximity and touch. J Fam Psychol [Internet]. 2003 [citado 24 abr 2019]; 17(1): 94-107. Disponible en: https:// psycnet.apa.org/record/2003-01658-009

14. Flacking R, Ewald U, Wallin L. Positive effect of Kangaroo Mother Care Long-Term Breastfeeding in very preterm infants. JOGNN [Internet]. 2011 [citado 27 mar 2019]; 40(2): 190-197. Disponible en: https://www.sciencedirect.com/science/article/ abs/pii/S088421751530530X?via\%3Dihub

15. Moore E, Bergman N, Anderson G y Medley N. Early skin-to-skin contact for mothers and their healthy newborn infants. Cochrane Database of Systematic Reviews [Internet]. 2016 [citado 6 jun 2019]; 11 (CD003519). Disponible en: 10.1002/14651858.CD003519.pub4

16. Ferreiro-Losada MT, Díaz-Sanisidroa E, Martínez-Romero MD, Rial-Boubeta A, VarelaMallou J, Clavería-Fontan A. Evaluación mediante grupos focales de las expectativas y percepciones de las mujeres durante el proceso del parto. RCA [Internet]. 2013 [citado 2 mar 2019]; 28(5): 265326. Disponible en: 10.1016/j.cali.2013.02.001

17. Lecannelier F, Ascanio L, Flores F, Hoffmann M. Apego \& Psicopatología: Una Revisión Actualizada Sobre los Modelos Etiológicos Parentales del Apego Desorganizado. Ter Psicol [Internet]. 2011 [citado 31 mar2020];29(1): 107-116. Disponible en: https:// pdfs.semanticscholar.org/1d51/cc6d54318268c6e 16d2689ab9172b71bd10a.pdf

18. Barg G. Bases neurobiológicas del apego. Revisión temática. Ciencias Psicológicas. [Internet]. 2011 [citado 2 mar 2019]; 5(1): 69-81. Disponible en: http://www.redalyc.org/articulo.oa?id=45954542 7007

19. Lucchini C, Márquez F, Uribe C. Efectos del contacto piel con piel del recién nacido con su madre. Index Enferm [Internet]. 2012 [citado 13 mar 2019]; 21(4): 209-213. Disponible en: http://scielo.isciii.es/scielo.php?script=sci_ arttext\&pid=S1132-12962012000300007

20. Acosta R, Piña C, Acosta L, López L. Método piel a piel: Evaluación del neurocomportamiento hasta el ańo de edad corregida. Rev Cubana Pediatr [Internet]. 2003 [citado 30 mar 2019]; 75(3). Disponible en: http://scielo.sld.cu/scielo.php?script =sci_arttext $\&$ pid $=$ S0034-75312003000300002 \&lng=es.

21. Arivabene J, Rubio MA. Método madre canguro: vivencias maternas y contribuciones para la enfermería. Rev Latino-Am Enfermagem [Internet]. 2010 [citado 12 abr 2019]; 18(2): 262268. Disponible en: http://www.scielo.br/scielo. php?pid=S0104 $-11692010000200018 \&$ script $=$ sc i_abstract\&tlng=es

22. Strauss A y Corbin J. Bases de la investigación cualitativa. Técnicas y procedimientos para desarrollar la teoría fundamentada [Internet]. $1^{\text {a }}$ ed. Español. Colombia: Editorial Universidad De Antioquía. 2002 [citado 2 dic 2018]. 354 p. Disponible en: https://diversidadlocal.files. wordpress.com/2012/09/bases-investigacioncualitativa.pdf

23. Flick U. Introducción a la Investigación Cualitativa [Internet]. Madrid: Editorial Morata S.L; 2004 [citado 2 dic 2018]: pp. 55-228. Disponible en: http://www.scielo.org.mx/pdf/redie/v7n $2 / v 7 n$ 2a10.pdf

24. Laise C, Carmen G, Margareth A. Vivendo no método canguru a tríade mãe-filho-família. Rev Latino-Am Enfermagem [Internet]. 2005 [citado 18 dic 2018]; 13(4): 562-568. Disponible en: 10.1590/S0104-11692005000400015.

25. Nubia C, Lucy M. Visión de las madres en el cuidado del hijo prematuro en el hogar. Av Enferm [Internet]. 2011 [citado 4 dic 2018]; 29(1): 120129. Disponible en: http://www.index-f.com/ $\mathrm{rae} / 291 / 120129 . \mathrm{php}$

26. Ocampo M. El hijo ajeno: vivencia de madres de niños prematuros hospitalizados. Aquichan [Internet]. 2013 [citado 4 dic 2018]; 13(1): 69-80 Disponible en: http://aquichan.unisabana.edu.co/ index.php/aquichan/article/view/2104

27. Sevilla-Godínez H, Sevilla-Godínez E. Consideraciones sobre el efecto de la presencia materna en la recuperación del recién nacido prematuro. Rev Sal Jal [Internet]. 2016 [citado 20 dic 2018]; 3(2): 93-95. Disponible en: https://www.medigraphic. com/pdfs/saljalisco/sj-2016/sj162g.pdf

28. Zambito S, Leash J, Baublitz W, Escher C, Shenberger MJ y Grim RD. Kangaroo Care: A Solution to Minimize Mother and Baby Separation. JOGNN [Internet]. 2010 [citado 26 ene 2019]; 39(1): 109. Disponible en: https:// www.jognn.org/article/S0884-2175(15)30364-6/ abstract

29. Reddy J, McInerney PA. The experiences of mothers who were implementing Kangaroo Mother Care (KMC) at a Regional Hospital in KwaZulu-Natal. Curationis [Internet]. 2007 [citado 12 mar 2019]; 30(3): 62-7. Disponible en: https://curationis.org. za/index.php/curationis/article/view/1104

30. Tessier R, Charpak N, Giron M, Cristo M, de Calume ZF, Ruiz-Peláez JG. Kangaroo Mother Care, home environment and father involvement in the first year of life: a randomized controlled study. Acta Paediatr [Internet]. 2009 [citado 8 abr 2019]; 98: 1444-1450. Disponible en: https:// www.ncbi.nlm.nih.gov/pubmed/19500083 
31. Silva L, Leite J, Scochi C, Sulva L, Silva T. La adhesión de las enfermeras al Método Canguro: Subvención para la administración del cuidado de enfermería. Rev Latino-Am Enfermagem [Internet]. 2015 [citado 7 abr 2019]; 23(3): 483490. Disponible en: http://www.scielo.br/pdf/ rlae/2015nahead/es_0104-1169-rlae-0339-2579. pdf

32. Dois A, Lucchini C, Villarroel L, Uribe C. Efecto del contacto piel con piel sobre la presencia de síntomas depresivos post parto en mujeres de bajo riesgo obstétrico. Rev chil pediatr [Internet]. 2013 [citado 28 dic 2018]; 84(3): 285-292. Disponible en: http://www.scielo. $\mathrm{cl} /$ scielo.php?script $=$ sci_arttext $\&$ pid $=$ S0370 41062013000300006\&lng=es

33. Tallandini MA, Scalembra C. Kangaroo Mother Care and Mother-Premature Infant Dyadic Interaction. IMH [Internet]. 2006 [citado 14 ene 2019]; 27(3): 251-275. Disponible en: https://onlinelibrary.wiley.com/doi/abs/10.1002/ imhj.20091

34. Cerón M, Argote L. La inasistencia al Programa Madre Canguro desde la teoría de la incertidumbre y del modelo Dreyfus. Av Enferm [Internet]. 2012 [citado 18 ene 2019]; (3): 70-82. Disponible en: https://revistas.unal.edu.co/index.php/avenferm/ article/view/39866

35. Hall D, Kirsten G. Kangaroo Mother Care - a review. Transfus Med [Internet]. 2008 [citado 24 ene 2019]; 18(2): 77-82. Disponible en: https:// www.ncbi.nlm.nih.gov/pubmed/18399840

36. Carbonell O, Plata S, Peña P. Calidad de cuidado materno: una comparación entre bebés prematuros en cuidado madre canguro y bebes a término en cuidado regular. Univ Psychol [Internet]. 2010 [citado 30 ene 2019]; 9(3): 773-785. Disponible en: http://webcache.googleusercontent.com/search? $\mathrm{q}=$ cache:Y9T3Pgsjq

37. Charpak N, Ruiz-Peláez JB. Resistance to implementing Kangaroo Mother Care in developing countries, and proposed solutions. Acta Paediatr [Internet]. 2006 [citado 20 ene 2019]; 95(5): 529-534. Disponible en: https://www.ncbi. nlm.nih.gov/pubmed/16825131

38. Ortiz A, Borré A, Carrillo S, Gutiérrez G. Relación de apego en madres adolescentes y sus bebés canguro. RLP [Internet]. 2006 [citado 4 ene 2019]; 38(1): 71-86. Disponible en: http://www. scielo.org.co/pdf/rlps/v38n1/v38n1a05.pdf

39. Rivara G, Rivara P, Cabrejos K, Quiñones E, Miñano K, Rusca F, et al. Contacto piel a piel inmediato: efecto sobre el estado de ansiedad y depresión materna posparto y sobre la adaptabilidad neonatal hacia la lactancia materna precoz. Rev peru pediatr [Internet]. 2007 [citado 30 ene 2019]; 60(3): 140-49. Disponible en: http:// sisbib.unmsm.edu.pe/BVRevistas/rpp/v60n3/pdf/ a02v60n3.pdf

40. Nguah SB, Wobil PN, Obeng R, Yakubu A, Kerber KJ, Lawn JE. Perception and practice of Kangaroo Mother Care after discharge from hospital in Kumasi, Ghana: A longitudinal study. BMC Pregnancy and Childbirth [Internet]. 2011 [citado 24 feb 2019]; 11: 1-8. Disponible en: https://www. ncbi.nlm.nih.gov/pubmed/22133462 